\title{
TRANSMISSÃO DA VOLATILIDADE ENTRE AÇÕES DE GRANDES E PEQUENAS EMPRESAS NO MERCADO BRASILEIRO DE CAPITAIS
}

\author{
VOLATILITY TRANSMISSION BETWEEN SMALL AND \\ LARGE CAPS IN THE BRAZILIAN MARKET
}

Recebido 09-05-2012 Aceito 26-05-2013

Marcelo Brutti Righi ${ }^{1}$ Paulo Sergio Ceretta ${ }^{2}$

\section{RESUMO}

Estudos acerca da transmissão de volatilidade entre ações de grandes e pequenas empresas vêm recebendo grande atenção nos últimos anos. Com base nessa ideia, o presente artigo visa analisar a ocorrência de transmissão de volatilidade entre pequenas e grandes empresas no Brasil. Para tanto, foram estimados modelos GARCH multivariados. Como proxies dos ativos, utilizaram-se os índices Small Cap (pequenas empresas) e Mid-Large (grandes empresas) da BM\&F/Bovespa. Os resultados permitem concluir que as ações de grandes empresas impactam a volatilidade condicional das ações das pequenas empresas, embora o contrário não seja verdadeiro. Esse resultado está de acordo com a literatura inerente ao tema abordado.

Palavras-chave: Transmissão de volatilidade, GARCH Multivariado, Grandes e Pequenas empresas.

\footnotetext{
${ }^{1}$ Possui graduação e mestrado em Administração pela Universidade Federal de Santa Maria - UFSM. Atualmente é doutorando em Administração pela Universidade Federal de Santa Maria - UFSM. Santa Maria, Rio Grande do Sul, Brasil. E-mail: marcelobrutti@hotmail. com.

${ }^{2}$ Possui graduação em Administração pela Universidade Federal de Santa Maria - UFSM, especialização em Estatística e Modelagem Quantitativa pela Universidade Federal de Santa Maria - UFSM, mestrado em Engenharia de Produção pela Universidade Federal de Santa Maria - UFSM e doutorado em Engenharia de Produção pela Universidade Federal de Santa Catarina - UFSC. Atualmente é professor associado da Universidade Federal de Santa Maria - UFSM. Santa Maria, Rio Grande do Sul, Brasil. E-mail: ceretta10@gmail.com.
} 


\section{ABSTRACT}

Researches about volatility transmission between small and large company stocks had received great attention in last years. Based on this idea, the current paper aims to analyze the occurrence of volatility transmission between small and large companies in Brazil. To that, we estimate multivariate GARCH models. As asset proxies we used the Small and Mid-Large cap BM\&F/Bovespa index. Results allow concluding that large company stocks impact the small stocks conditional volatility. However, the relationship does not maintain it in reverse direction. This result is in accord with the addressed subject.

Keywords: Volatility Spillover, Multivariate GARCH, Large and Small Companies.

\section{INTRODUÇÃO}

O acompanhamento e a gestão de grandes ativos financeiros são rotina para muitos indivíduos e organizações. Consequentemente, a análise cuidadosa, a especificação, estimativa e a previsão da dinâmica de retornos dos ativos financeiros, bem como a construção e a avaliação das carteiras, são habilidades essenciais no conjunto de ferramentas de qualquer planejador financeiro e analista (CAPORIN e MCALEER, 2010).

Inserido nesse contexto, o conhecimento do comportamento estocástico de correlações e covariâncias entre retornos de ativos é parte essencial à precificação de ativos, seleção de carteira e administração do risco (BAUR, 2006). Dessa forma, o estudo da volatilidade assume grande importância na área de finanças, em especial no apreçamento de derivativos e no gerenciamento de risco de investimentos. Tradicionalmente, o cálculo de estimativas da volatilidade de retornos financeiros, bem como a sua aplicação na determinação do valor em risco (value at risk, VaR), toma como base as variações diárias nos preços dos ativos (GOODHART e O'HARA, 1997).

Nesse sentido, a literatura acerca dos modelos de mensuração de volatilidade inclui diversos métodos com distintos graus de dificuldade de implementação. De um ponto de vista geral, o estimador mais simples é o desvio padrão histórico, que atribui peso uniforme a todas as observações. A vantagem desse estimador consiste na facilidade de cálculo e interpretação; seus inconvenientes são sua simetria e o fato de ser constante. Em contraste, os modelos ARCH (autoregressive conditional heteroscedasticity), introduzidos por Engle (1982), e sua generalização GARCH (generalized autoregressive conditional heteroscedasticity), proposta por Bollerslev (1986), por não sofrerem desses problemas, são mais amplamente aplicados para modelar a volatilidade de séries financeiras. Não obstante, muitas variações da modelagem GARCH têm sido propostas, como, por exemplo, o modelo GARCH exponencial (EGARCH), pensado por Nelson (1991). Este modelo permite a inclusão de efeitos assimétricos da volatilidade condicional.

Embora correlações incondicionais possam ser facilmente estimadas, o mesmo não ocorre com correlações estocásticas. Apesar disso, é possível estender os conceitos acerca da mensuração da volatilidade condicional para uma perspectiva multivariada, por meio de um modelo GARCH multivariado (MVGARCH). Esse tipo de modelo é bastante interessante porque permite identificar e utilizar movimentos comuns entre volatilidades de ativos distintos.

Apesar desta capacidade, quando a análise da volatilidade se volta para estudos envolvendo mais de um ativo, a relevância de uma possível influência do mercado externo mais robusto é frequentemente ignorada (DE LUCA, GENTON e LOPERFIDO, 2006). Não obstante, esse é um importante tema que pode auxiliar na explicação de algumas questões empíricas. Nesse sentido, uma ampla literatura tem abordado a questão, tratando da transmissão de volatilidade entre ativos distintos.

Fundamentando-se nesse raciocínio, Karmakar (2008) enfatiza que mecanismos de transmissão dos retornos e volatilidade entre grandes e pequenas empresas têm recebido aten- 
ção de acadêmicos e profissionais da área, devido a suas implicações para a gestão de carteiras, bem como para a precificação de ativos. Tal relevância se deve, em grande parte, ao fato de que uma série de estudos tem demonstrado que essas transmissões são assimétricas, isto é, que os retornos de carteiras de ações pequenas tendem a ser correlacionados com os retornos defasados de carteiras de ações de grande porte, enquanto os retornos de carteiras formadas por ações de grandes empresas tendem a não ser correlacionados com os retornos defasados de pequenas carteiras formadas por ações de empresas menores.

Assim, o presente artigo possui como objetivo primordial verificar a ocorrência de transmissão de volatilidade entre pequenas e grandes empresas no Brasil. Para tanto, aplicar-se-á o método GARCH multivariado com modelos de correlação condicional dinâmica (DCC) e correlação condicional constante (CCC). Como proxies das empresas grandes e pequenas, serão utilizados os índices Small cap e Mid-Large cap da Bolsa de Valores, Mercadorias e Futuros de São Paulo (BM\&F/Bovespa).

O restante do artigo está estruturado da seguinte forma: a seção 2 apresenta uma sucinta revisão teórica acerca da transmissão de volatilidade entre grandes e pequenas empresas, assim como os métodos empregados para sua verificação; a seção 3 expõe os aspectos metodológicos utilizados na presente pesquisa; a seção 4 contém os resultados obtidos, assim como sua análise; e a seção 5 traz as conclusões do estudo em questão.

\section{MODELAGEM DA VOLATILIDADE}

Modelagem e previsão da dinâmica multidimensional da volatilidade de um conjunto de ativos são pré-requisitos muito importantes para auxiliar as tomadas de decisões e ações financeiras, tais como construção e avaliação de uma carteira ótima, determinação de preços de produtos estruturados que são projetados com mais de um ativo subjacente ou mesmo cálculo de uma razão de cobertura. Uma enorme variedade de métodos para obter estimativas confiáveis das correlações entre variáveis financeiras têm sido desenvolvida, incluindo modelos simples (sem estimadores históricos) e métodos sofisticados (que tentam encontrar aproximações razoáveis para os processos estocásticos e distribuições condicionais que conduzem e determinam a dinâmica conjunta de volatilidades de ativos) (PUTINTSEVA, 2010).

Consequentemente, modelos multivariados de volatilidade têm atraído um interesse considerável durante a última década. Essa situação pode estar associada com o aumento da disponibilidade de dados financeiros, o incremento da capacidade de processamento dos computadores e o fato de que o setor financeiro começou a perceber as possíveis vantagens desses modelos.

Desde Lo e MacKinlay (1990a; 1990b), muitos estudos têm documentado a dinâmica temporal entre os retornos das ações das grandes e pequenas empresas (Mech, 1993; McQueen, Pinegar e Thorley, 1996; Campbell, Lo e MacKinlay, 1997). Tais autores descobriram que há correlações cruzadas entre retornos das ações de grandes e pequenas empresas. Além disso, uma série de estudos também tem demonstrado que essas correlações cruzadas são assimétricas, ou seja, que os retornos de carteiras de ações pequenas tendem a estar correlacionados com os retornos defasados de carteiras de ações de grande porte, enquanto os retornos de carteiras compostas por ações de grandes empresas tendem a não apresentar correlação com os retornos defasados das carteiras de ações das pequenas empresas.

Corroborando essa ideia, Lo e Mackinlay (1990a) relatam que alterações de preço em ações das grandes empresas tendem a impactar as ações das pequenas empresas, mas que o inverso não ocorre. Esses autores excluíram a falta de sincronia nas negociações como uma possível explicação para as correlações cruzadas. Dessa forma, uma série de outras explicações foi proposta 
(KARMAKAR, 2008). Não obstante, Mech (1993) sugere que a assimetria na correlação cruzada entre os retornos das ações de grandes e pequenas empresas é devida aos custos de transação e mostra que a velocidade de ajustamento dos preços é superior em grandes empresas.

Grinblatt, Titman e Wermers (1995) argumentam que repercussões assimétricas nos retornos das ações das grandes e pequenas empresas estão relacionadas aos padrões assimétricos de negociação e ao comportamento dos investidores institucionais. Conrad, Gultekin e Kaul (1991) destacam que a assimetria existente na transmissão dos retornos (horizontes curtos de tempo) entre grandes e pequenas ações no EUA também ocorre em relação à volatilidade. Estes autores enfatizam que os choques de volatilidade das grandes ações impactam a volatilidade futura das ações das empresas de pequeno porte, mas que choques de volatilidade das ações menores tem pouco ou nenhum impacto sobre a volatilidade futura das grandes ações.

Uma vez que a volatilidade do preço das ações está diretamente relacionada ao fluxo de informações do mercado (ROSS, 1989), a assimetria na transmissão de volatilidade entre as ações das grandes e pequenas empresas é consistente com um mercado em que preços de grandes ações respondem às novas informações imediatamente, enquanto os preços das pequenas ações respondem com certo atraso.

Assim, uma vez que o fluxo de informações deve ser assimétrico entre grandes e pequenas empresas, a transmissão de volatilidade também é assimétrica. Tal explicação é fundamentada por McQueen, Piegar, e Thorley (1996), que enfatizam que pequenas ações, no momento em que sua notícia chega ao mercado, apresentam uma reação atrasada, em comparação com grandes ações.

Dessa forma, testes empíricos da teoria acerca das repercussões de volatilidade, baseada na modelagem GARCH, foram introduzidos pela primeira vez por Engle, Ito e Lin (1990). De acordo com Tsay (2005), um modelo $\operatorname{GARCH}(p, q)$ pode ser definido conforme a equação [1].

$$
\begin{aligned}
& \sigma_{t}^{2}=\alpha_{0}+\sum_{i=1}^{p} \alpha_{i} a_{t-i}^{2}+\sum_{j=1}^{q} \beta_{j} \sigma_{t-j}^{2} ; \\
& a_{t}=\sigma_{t} \epsilon_{t} .
\end{aligned}
$$

Na formulação [1], $\sigma_{t}^{2}$ é a variância de uma série no instante $t, a_{t}$ é a inovação (choque) sobre o retorno de uma série no instante $t,\left\{\epsilon_{t}\right\}$ é uma sequência de variáveis aleatórias independentes e identicamente distribuídas com média zero e variância igual à unidade, $\alpha_{i}$ e $\beta_{j}$ são parâmetros, de tal forma que $\alpha_{0}>0, \alpha_{i} \geq 0, \beta_{j} \geq 0$ e $\sum_{\mathrm{i}=1}^{\max (\mathrm{p}, \mathrm{q})}\left(\alpha_{\mathrm{i}}+\beta_{\mathrm{i}}\right)<1$. Essa última restrição tem como implicação o fato de que a variância incondicional de $a_{t}$ é finita, isto é, sua variância condicional evolui com o tempo.

Entretanto, conforme Dark, Zhang e Qu (2010), para examinar os padrões de fluxos de informação entre diferentes mercados de ações, é preciso que seja empregado um modelo GAR$\mathrm{CH}$ multivariado. Dessa forma, Bollerslev (1986) enfatiza que, comparado a um modelo GARCH univariado, o modelo multivariado utiliza informações de duas ou mais séries históricas de preços distintas e analisa as repercussões da volatilidade entre elas. Com base nessa ótica, Chan, Chan, e Karolyi (1991) fazem uma discussão detalhada a respeito da necessidade de focar em transmissões de volatilidade entre os mercados de ações spot (à vista) e futuro.

Porém, quando se trata da especificação de modelo GARCH multivariado, existe um dilema. Por um lado, o modelo deve ser flexível o suficiente para ser capaz de representar a dinâmica das variâncias e covariâncias condicionais. Por outro lado, como o número de parâmetros em um modelo GARCH multivariado aumenta rapidamente com a sua dimensão, a especificação deve ser parcimoniosa o suficiente para permitir a estimação do modelo com relativa facilidade, assim como consentir uma simples interpretação dos seus parâmetros (SILVENNOINEN e TERÄSVISTRA, 
2008). Contudo, parcimônia, muitas vezes, significa simplificação, e modelos com apenas alguns parâmetros podem não ser capazes de capturar a dinâmica relevante na estrutura de covariância.

Com base nisso, outra característica que precisa ser levada em conta na especificação é a restrição de positividade (matrizes de covariância devem necessariamente ter seus determinantes definidos como positivos). Uma possibilidade é obter condições para que os determinantes das matrizes de covariância condicional, implícitas no modelo, sejam definidos como positivos, mas esse procedimento é muitas vezes inviável na prática. Uma alternativa é formular o modelo de forma que a positividade seja decorrente da estrutura especificada (em adição a algumas restrições simples).

Tendo por fundamento essa ideia, considera-se o modelo $G A R C H$ multivariado com parametrização $\mathrm{VECH}$, representado pela equação [2].

$$
\operatorname{vech}\left(H_{t}\right)=A_{0}+\sum_{j=1}^{q} \beta_{j} \operatorname{vech}\left(H_{t-1}\right)+\sum_{j=1}^{p} A_{j} \operatorname{vech}\left(\varepsilon_{t-j}, \varepsilon_{t-j}^{\prime}\right) \text {. }
$$

$\mathrm{Na}$ formulação [2], vech é o operador que contém o triângulo inferior de uma matriz simétrica em um vetor, $H_{t}$ descreve a variância condicional e o termo de erro é $\varepsilon_{t}=H_{t}^{1 / 2} \eta_{t}, \eta_{t} \sim i i d N(0,1)$. A desvantagem desse modelo é seu grande número de parâmetros. Além disso, visando garantir a positividade de $H_{t}$, restrições precisam ser impostas.

Dessa forma, emerge como alternativa a parametrização $B E K K$, sugerida por Engle e Kroner (1995). A parametrização $B E K K$, que cuida essencialmente dos problemas anteriormente mencionados acerca do modelo $V E C H$, é definida como exibido em [3].

$$
H_{t+1}=C^{\prime} C+B^{\prime} H_{t} B+A^{\prime} \epsilon_{t} \epsilon_{t}^{\prime} \text {. }
$$

As matrizes $A, B$ e $C$, que contém os coeficientes para o caso com dois ativos, são definidas como:

$$
\begin{aligned}
& A=\left[\begin{array}{ll}
a_{11} & a_{12} \\
a_{21} & a_{22}
\end{array}\right] \\
& B=\left[\begin{array}{ll}
b_{11} & b_{12} \\
b_{21} & b_{22}
\end{array}\right] \\
& C=\left[\begin{array}{cc}
c_{11} & c_{12} \\
0 & c_{22}
\end{array}\right]
\end{aligned}
$$

Em [3], $H_{t+1}$ é uma matriz de covariância condicional. No caso bivariado, o parâmetro $B$ explica a relação entre as variâncias condicionais passadas e a atual (GARCH). O parâmetro $A$ mede a extensão em que as variâncias condicionais estão correlacionadas com os erros passados ao quadrado, ou seja, ele capta os efeitos de choques ou volatilidade $(A R C H)$.

$\mathrm{O}$ número total de parâmetros estimados é 11 . No caso da parametrização $B E K K$, as volatilidades da equação [3] possuem as formas [5] e [6].

$$
\begin{aligned}
h_{11, t+1}= & c_{11}^{2}+b_{11}^{2} h_{11, t}+2 b_{11} b_{12} h_{12, t}+b_{21}^{2} h_{22, t}+a_{11}^{2} \varepsilon_{1, t}^{2}+2 a_{11} a_{12} \varepsilon_{1, t} \varepsilon_{2, t}+a_{21}^{2} \varepsilon_{2, t}^{2} \\
\mathrm{~h}_{22, \mathrm{t}+1}= & \mathrm{c}_{12}^{2}+\mathrm{c}_{22}^{2}+\mathrm{b}_{12}^{2} \mathrm{~h}_{11, \mathrm{t}}+2 \mathrm{~b}_{12} \mathrm{~b}_{22} \mathrm{~h}_{12, \mathrm{t}}+\mathrm{b}_{22}^{2} \mathrm{~h}_{22, \mathrm{t}}+\mathrm{a}_{12}^{2} \varepsilon_{1, \mathrm{t}}^{2}+2 \mathrm{a}_{12} \mathrm{a}_{22} \varepsilon_{1, \mathrm{t}} \varepsilon_{2, \mathrm{t}}+ \\
& \mathrm{a}_{22}^{2} \varepsilon_{2, \mathrm{t}^{*}}^{2}
\end{aligned}
$$

As equações [5] e [6] evidenciam como os choques de volatilidade são transmitidos ao longo do tempo através dos índices. Tais equações são estimadas para um par de séries de cada vez. Assumindo que os erros são normalmente distribuídos e buscando estimar os parâmetros do modelo proposto, maximiza-se, através do algoritmo BHHH (Berndt, Hall, Hall e Hausman), a função de verossimilhança, representada por [7].

$$
L(\theta)=-T \ln (2 \pi)-\frac{1}{2} \sum_{t=1}^{T} \ln \left(\left|H_{t}\right|+\varepsilon_{t}^{\prime} H_{t}^{-1} \varepsilon_{t}\right) .
$$


Em [7], $\vartheta$ é o vetor de parâmetros estimados e $T$ é o número de observações. Engle e Kroner (1995) sugeriram que esse algoritmo seja empregado para obter as condições iniciais desejadas.

Todavia, o modelo de parametrização BEKK possui como desvantagem a difícil interpretação de seus parâmetros estimados. As formulações [5] e [6] evidenciam que, mesmo para o caso de modelagem bivariada, a interpretação dos coeficientes pode ser confusa, pois não há parâmetros que são governados exclusivamente por uma equação (BAUR, 2006). Sendo assim, é possível que um determinado parâmetro estimado possua viés devido ao fato de o mesmo influenciar duas equações simultaneamente (TSE, 2000).

Dessa forma, conforme enfatizado por Peters (2008), uma abordagem para contornar o problema da interpretação dos parâmetros é o modelo de matriz de covariância condicional, observada indiretamente através da matriz de correlações condicionais. O primeiro modelo desse tipo foi o de correlação condicional constante (CCC), proposto por Bollerslev (1992). A correlação condicional foi assumida como constante, e somente os desvios condicionais são variáveis no tempo. O modelo CCC pode ser definido conforme a formulação [8].

$$
H_{t}=D_{t} R D_{t}=\left(\rho_{i j} \sqrt{h_{i i, t} h_{j j, t}}\right)
$$

Na formulação [8], $D_{t}=\operatorname{diag}\left(h_{11, t}^{1 / 2} \ldots h_{N N, t}^{1 / 2}\right)$, em que $h_{i i, t}$ é definido de maneira similar a qualquer modelo GARCH univariado, $R=\left(\rho_{i j}\right)$ é uma matriz simétrica positiva definida, $\operatorname{com} \rho_{i i}=1, \forall i$, ou seja, $R$ é a matriz que contém as correlações condicionais constantes $\rho_{i j}$.

O modelo CCC original possui uma especificação no formato $\operatorname{GARCH}(1,1)$ para cada variância condicional em $D_{t}$, como representado pela formulação [9].

$$
h_{i i, t}=\omega_{i}+\alpha_{i} \varepsilon_{i, t-1}^{2}+\beta_{i} h_{i i, t-1}, i=1, \ldots, N
$$

Esse modelo CCC possui $N(N+5) / 2$ parâmetros. $H_{t}$ é definida positiva se e somente se todas as $N$ variâncias condicionais são positivas e $R$ é definida positiva. As variâncias incondicionais são facilmente obtidas, assim como no modelo GARCH univariado, porém as covariâncias incondicionais são difíceis de calcular, devido à não linearidade de [8].

Entretanto, conforme Bauwens, Laurent e Rombouts (2003), a suposição de que a correlação condicional é constante ao longo do tempo não é convincente, uma vez que, na prática, a correlação entre ativos sofre muitas mudanças ao longo do tempo. Assim sendo, Engle e Sheppard (2001) introduzem o modelo de correlação condicional dinâmica (DCC). O modelo DCC tem um algoritmo de dois passos para estimar os parâmetros que o tornam relativamente simples de usar na prática. Na primeira etapa, a variância condicional é estimada via modelo GARCH univariado para cada ativo. Na segunda etapa, os parâmetros para a correlação condicional, tendo em vista os parâmetros da primeira etapa, são estimados. Finalmente, o modelo DCC inclui condições que tornam a matriz de covariância definida positiva em todos os pontos no tempo e a covariância do processo estacionária. O modelo DCC é representado pela formulação [10].

$$
\begin{aligned}
H_{t}= & D_{t} R_{t} D_{t} . \\
& \text { Onde: } \\
R_{t}= & \operatorname{diag}\left(q_{11, t}^{-1 / 2} \ldots q_{N N, t}^{-1 / 2}\right) Q_{t} \operatorname{diag}\left(q_{11, t}^{-1 / 2} \ldots q_{N N, t}^{-1 / 2}\right) .
\end{aligned}
$$

Sendo que a matriz quadrada de ordem $N$ simétrica positiva que define $Q_{t}=\left(q_{i j, t}\right)$ possui a formulação proposta em [12].

$$
Q_{t}=(1-\alpha-\beta) \bar{Q}+\alpha u_{t-1} u_{t-1}^{\prime}+\beta Q_{t-1}
$$


Na formulação [12], $u_{i, t}=\varepsilon_{i, t} / \sqrt{h_{i, t}} ; \bar{Q}$ é a matriz $N \times N$ composta pela variância incondicional de $u_{t ;} \alpha$ e $B$ são parâmetros escalares não negativos, satisfazendo a relação $\alpha+\beta<1$.

\section{ASPECTOS METODOLÓGICOS}

Para verificar a ocorrência de transmissão de volatilidade entre grandes e pequenas empresas no Brasil, foram coletados dados referentes ao nível em pontos dos índices Small cap e Mid-Large cap da BM\&F/Bovespa, compreendendo o período de 01/09/2008 a 19/04/2010, totalizando 377 observações para cada indicador. A escolha desses índices para proxies do desempenho das grandes e pequenas empresas ocorreu pelo fato de terem sido criados com o objetivo de medir o comportamento das empresas listadas na BM\&F/Bovespa de modo segmentado, com o índice Mid-Large medindo o retorno de uma carteira composta pelas empresas listadas de maior capitalização e o índice Small Cap medindo o retorno de uma carteira composta por empresas de menor capitalização. As ações componentes são selecionadas por sua liquidez e são ponderadas nas carteiras pelo valor de mercado das ações disponíveis à negociação.

Através do nível em pontos dos índices estudados, foi calculada a variação do logaritmo natural das séries, visando a sua utilização para auferir a transmissão de volatilidade entre os indicadores. Posteriormente, por meio do teste $Q$ de Ljung e Box (1978), representado pela formulação [13], que testa a hipótese nula de que os dados são aleatórios contra a alternativa de não aleatoriedade dos mesmos, procurou-se identificar a presença de correlação serial na série dos retornos dos índices.

$$
Q=n(n+2) \sum_{k=1}^{h} \frac{\hat{\rho}_{k}^{2}}{n-k}
$$

Na formulação [13], $n$ é o tamanho da amostra; $\hat{\rho}_{k}^{2}$ é a autocorrelação da amostra na defasagem $k ; h$ é o número de defasagens sendo testadas. A estatística $Q$ de Ljung e Box segue a distribuição de qui-quadrado $\left(\chi^{2}\right)$.

Uma vez que a série apresenta dependência serial, os resultados obtidos podem conter vieses de estimação. Dessa forma, conforme exposto por Karmakar (2008), para possibilitar a filtragem da dependência serial dos resíduos estimados, será utilizado um vetor autorregressivo (VAR) visando obter a estimativa média da série de retorno de cada índice. A forma matemática da modelagem VAR utilizada é representada pela equação [14].

$$
\operatorname{VAR}(s, l)=\left\{\begin{array}{l}
\Delta s_{t}=\beta_{0}+\sum_{i=1}^{n} \beta_{i} \Delta s_{t-i}+\sum_{j=1}^{m} \beta_{j} \Delta l_{t-j}+\varepsilon_{1, t} \\
\Delta l_{t}=\alpha_{0}+\sum_{i=1}^{n} \alpha_{i} \Delta s_{t-i}+\sum_{j=1}^{m} \alpha_{j} \Delta s_{t-j}+\varepsilon_{2, t}
\end{array}\right.
$$

Na formulação [14], $\Delta s_{t}$ e $\Delta l_{t}$ são os retornos sobre os índices Small cap e Mid-Large Cap, respectivamente; $\alpha_{q}$ e $\beta_{q}$ são parâmetros de regressão, $q=1, \ldots, m, \ldots n ; \varepsilon_{1, t}$ e $\varepsilon_{2, t}$ são os resíduos da série de retornos estimados.

Dessa forma, para escolher o número de defasagens empregadas na estimação do modelo VAR, foram aplicados o logaritmo de verossimilhança (LL), o critério de informação de Akaike (AIC), o critério de informação Bayesiano (BIC) e o critério de informação de Hannan-Quinn (HQC) (AKAIKE, 1973; AKAIKE, 1974; SCHWARZ, 1978; HANNAN e QUINN, 1979). As representações matemáticas de tais critérios estão expostas nas formulações [15], [16] e [17], respectivamente.

$$
\begin{aligned}
& A I C=\log \left[\frac{1}{N} \sum_{i=1}^{N}\left(y_{t}-\hat{y}_{t}\right)^{2}\right]+\frac{2}{k} N \\
& B I C=\log \left[\frac{1}{N} \sum_{i=1}^{N}\left(y_{t}-\hat{y}_{t}\right)^{2}\right]+\frac{k}{N} \log (N) \\
& H Q C=\log \left[\frac{1}{N} \sum_{i=1}^{N}\left(y_{t}-\hat{y}_{t}\right)^{2}\right]+2 k \ln [\ln (N)]
\end{aligned}
$$


Assim, $k$ é o número de parâmetros no modelo; $N$ é o nímero de observações; $y_{t}$ é o valor observado da variável dependente do modelo no tempo $t ; \hat{y}_{t}$ é o valor estimado da variável dependente do modelo no instante $t$. Não obstante, após estimação do modelo VAR, será realizada a verificação da significância conjunta de suas defasagens por meio de teste $F$.

Posteriormente a essa análise empírica inicial, com base nos resíduos que serão obtidos por meio de [14], serão utilizados os modelos GARCH multivariado CCC e DCC, expostos anteriormente no presente artigo. Tal aplicação visará identificar a magnitude da transmissão de volatilidade entre as ações de grandes e pequenas empresas no Brasil.

\section{ANÁLISE DOS RESULTADOS}

A fim de identificar a presença de correlação serial nas séries compostas pela variação do logaritmo natural dos índice, foram calculadas estatísticas $Q$ de Ljung e Box para ambas as séries. Os resultados estão expostos nas Tabelas 1 e 2, para os índices Small Cap e Mid-Large Cap, respectivamente.

Como pode ser auferido, baseado nos resultados presentes na Tabela 1, verificou-se ocorrência de correlação serial na variação dos logaritmos do índice Small Cap, com ênfase para as defasagens 1,3 e 12, as quais detêm estatística $Q$ de Ljung e Box significante ao nível de $5 \%$, assim como valores significativos para as funções de autocorrelação (ACF) e autocorrelação parcial (PACF). Analogamente, a Tabela 2 contém os resultados para as funções de autocorrelação e autocorrelação parcial, assim como a estatística $Q$ de Ljung e Box calculada para a série composta pelas variações dos logaritmos do índice Mid-Large Cap, no intuito de verificar a ocorrência de dependência serial nessa série.

Tabela 1. Função de autocorrelação, função de autocorrelação parcial e estatística $Q$ das defasagens da série de variações dos logaritmos do índice Small Cap diário.

\begin{tabular}{ccccccc}
\hline Defasagem & ACF & & PACF & & Q-stat, & {$[p$-valor $]$} \\
\hline 1 & 0,119 & $* *$ & 0,119 & $* *$ & 5,740 & {$[0,017]$} \\
2 & $-0,008$ & & $-0,022$ & & 5,765 & {$[0,056]$} \\
3 & $-0,082$ & & $-0,079$ & & 8,466 & {$[0,037]$} \\
4 & 0,158 & $* * *$ & 0,181 & $* * *$ & 18,677 & {$[0,001]$} \\
5 & $-0,032$ & & $-0,080$ & & 19,085 & {$[0,002]$} \\
6 & $-0,038$ & & $-0,030$ & & 19,686 & {$[0,003]$} \\
7 & 0,013 & & 0,057 & & 19,753 & {$[0,006]$} \\
8 & 0,067 & & 0,018 & & 21,613 & {$[0,006]$} \\
9 & 0,032 & & 0,033 & & 22,043 & {$[0,009]$} \\
10 & $-0,013$ & & $-0,005$ & & 22,112 & {$[0,015]$} \\
11 & $-0,061$ & & $-0,069$ & & 23,645 & {$[0,014]$} \\
12 & 0,195 & $* * *$ & 0,221 & $* * *$ & 39,508 & {$[0,000]$} \\
\hline$* *$
\end{tabular}

**significativo ao nível de $5 \%, * * *$ significativo ao nível de $1 \%$.

Tabela 2. Função de autocorrelação, função de autocorrelação parcial e estatística $Q$ das defasagens da série de variações dos logaritmos do índice Small Cap diário.

\begin{tabular}{ccccccc}
\hline Defasagem & ACF & & PACF & & Q-stat, & {$[p$-valor $]$} \\
\hline 1 & 0,062 & & 0,062 & & 1,560 & {$[0,212]$} \\
2 & $-0,114$ & $* *$ & $-0,118$ & $* *$ & 6,799 & {$[0,033]$} \\
3 & $-0,126$ & $* *$ & $-0,112$ & $* *$ & 13,190 & {$[0,004]$}
\end{tabular}




\begin{tabular}{ccccccc}
\hline Defasagem & ACF & & PACF & & Q-stat, & {$[p$-valor $]$} \\
\hline 4 & 0,127 & $* *$ & 0,132 & $* * *$ & 19,709 & {$[0,001]$} \\
5 & $-0,085$ & $*$ & $-0,135$ & $* * *$ & 22,682 & {$[0,000]$} \\
6 & $-0,078$ & & $-0,052$ & 25,156 & {$[0,000]$} \\
7 & $-0,032$ & & $-0,013$ & 25,586 & {$[0,001]$} \\
8 & 0,051 & & $-0,005$ & & 26,642 & {$[0,001]$} \\
9 & 0,034 & 0,039 & 27,116 & {$[0,001]$} \\
10 & 0,000 & & $-0,001$ & & 27,116 & {$[0,002]$} \\
11 & $-0,138$ & $* * *$ & $-0,143$ & $* * *$ & 35,037 & {$[0,000]$} \\
12 & 0,070 & 0,095 & $*$ & 37,065 & {$[0,000]$} \\
\hline
\end{tabular}

Dessa forma, tendo por base os resultados da Tabela 2, verificou-se a presença de correlação serial na série composta pelas variações dos logaritmos do índice Mid-Large Cap, enfatizando as defasagens $2,3,4,5,11$, as quais obtiveram, além de estatística $Q$ de Ljung Box significante, ACF e PACF estatisticamente significativas.

Os resultados apresentados nas Tabelas 1 e 2 evidenciam o fato de que as observações das séries das variações dos logaritmos dos índices presentes neste estudo não são aleatórias e sim dependentes serialmente das observações defasadas. Tal resultado implica o fato de que estimativas dos parâmetros visando auferir a ocorrência de transmissão de volatilidade entre ações de empresas grandes e pequenas estariam enviesadas. Assim sendo, passou a ser preciso estimar um modelo VAR, com o intuito de filtrar tais dependências seriais. Dessa forma, foi necessário definir a ordem de defasagem do modelo VAR a ser estimado. Para tanto, foram empregues o logaritmo de máxima verossimilhança e os critérios de informação de Akaike, Bayesiano e Hannan-Quinn. Os resultados calculados para definir a ordem de defasagem do modelo VAR estão contidos na Tabela 3.

Tabela 3. Verificando o número de defasagens a ser utilizada no VAR, com base nos critérios LL, AIC, BIC e HQC.

\begin{tabular}{cccccc}
\hline Defasagem & $\mathrm{LL}$ & $\mathrm{p}(\mathrm{LR})$ & $\mathrm{AIC}$ & $\mathrm{BIC}$ & $\mathrm{HQC}$ \\
\hline 1 & $\mathbf{2 1 5 6 , 1 9 1}$ & & $-11,055$ & $-\mathbf{1 0 , 9 9 4}$ & $\mathbf{- 1 1 , 0 3 1}$ \\
2 & 2162,581 & 0,012 & $-11,067$ & $-10,965$ & $-11,027$ \\
3 & 2166,690 & 0,084 & $-11,068$ & $-10,925$ & $-11,011$ \\
4 & 2172,805 & 0,016 & $-11,079$ & $-10,895$ & $-11,006$ \\
5 & 2176,942 & 0,082 & $-11,079$ & $-10,855$ & $-10,991$ \\
6 & 2178,584 & 0,511 & $-11,067$ & $-10,802$ & $-10,962$ \\
7 & 2182,454 & 0,102 & $-11,067$ & $-10,761$ & $-10,945$ \\
8 & 2185,250 & 0,232 & $-11,060$ & $-10,714$ & $-10,923$ \\
9 & 2188,252 & 0,199 & $-11,055$ & $-10,668$ & $-10,902$ \\
10 & 2190,938 & 0,251 & $-11,049$ & $-10,621$ & $-10,879$ \\
11 & 2198,782 & 0,003 & $-11,068$ & $-10,600$ & $-10,882$ \\
12 & $\mathbf{2 2 1 3 , 3 6 2}$ & $\mathbf{0 , 0 0 0}$ & $-\mathbf{1 1 , 1 2 3}$ & $-10,613$ & $-10,921$ \\
\hline
\end{tabular}

É possível auferir, com base na Tabela 3, que LL e AIC possuem valores que indicam que 12 defasagens devem formar o modelo VAR, enquanto que BIC e HQC apontam para a utilização de uma defasagem. Não obstante, devido à maior significância do logaritmo de verossimilhança, optou-se por estimar um modelo VAR contendo 12 defasagens para as variações dos logaritmos de cada índice. 
Após a estimação do modelo VAR com 12 defasagens, empregou-se o teste $F$ a fim de verificar a significância conjunta dos parâmetros desse modelo. Os resultados estão expostos na Tabela 4. Com base na Tabela 4, fica nítido que os parâmetros das defasagens do modelo VAR estimado são significativas em conjunto, como evidenciam os valores calculados para os testes $F$ executados, bem como suas respectivas significâncias.

Para referenciar ainda mais o modelo VAR estimado, que possui como intuito filtrar a dependência serial, são exibidos, nas Tabelas 5 e 6, ACF, PACF e estatística $Q$ de Ljung e Box das defasagens dos resíduos das séries dos índices Small Cap e Mid-Large Cap, respectivamente.

Tabela 4. Testes-F para verificar a significância conjunta das defasagens no VAR(12)
$\begin{array}{cccc}\text { Todas as defasagens do Índice Small Cap } & F(12,364)=3,151 & {[0,0003]} \\ \text { Todas as defasagens do Índice Mid-Large Cap } & F(12,364)=3,025 & {[0,0005]} \\ \text { Todas as variáveis, 12 defasagens } & F(2,364)=11,645 & {[0,0000]}\end{array}$

Tabela 5. Função de autocorrelação, função de autocorrelação parcial e estatística $Q$ para a série de resíduos do índice Small Cap estimados pelo VAR(12).

\begin{tabular}{ccccc}
\hline Defasagem & ACF & PACF & $\begin{array}{c}\text { Q-s- } \\
\text { tat, }\end{array}$ & [p-valor] \\
\hline 1 & $-0,018$ & $-0,018$ & 0,127 & {$[0,721]$} \\
2 & $-0,020$ & $-0,020$ & 0,281 & {$[0,869]$} \\
3 & $-0,019$ & $-0,020$ & 0,429 & {$[0,934]$} \\
4 & 0,016 & 0,015 & 0,525 & {$[0,971]$} \\
5 & $-0,010$ & $-0,010$ & 0,560 & {$[0,990]$} \\
6 & 0,018 & 0,018 & 0,695 & {$[0,995]$} \\
7 & $-0,027$ & $-0,026$ & 0,974 & {$[0,995]$} \\
8 & 0,010 & 0,009 & 1,011 & {$[0,998]$} \\
9 & $-0,040$ & $-0,040$ & 1,655 & {$[0,996]$} \\
10 & $-0,012$ & $-0,014$ & 1,710 & {$[0,998]$} \\
11 & 0,027 & 0,026 & 1,997 & {$[0,999]$} \\
12 & 0,012 & 0,010 & 2,052 & {$[0,999]$} \\
\hline
\end{tabular}

Tabela 6. Função de autocorrelação, função de autocorrelação parcial e estatística $Q$ para a série de resíduos do índice Mid-Large Cap estimados pelo VAR(12).

\begin{tabular}{ccccc}
\hline Defasagem & ACF & PACF & Q-stat, & {$[p$-valor $]$} \\
\hline 1 & $-0,008$ & $-0,008$ & 0,024 & {$[0,878]$} \\
2 & $-0,030$ & $-0,030$ & 0,375 & {$[0,829]$} \\
3 & $-0,007$ & $-0,007$ & 0,393 & {$[0,942]$} \\
4 & 0,028 & 0,027 & 0,697 & {$[0,952]$} \\
5 & 0,001 & 0,001 & 0,697 & {$[0,983]$} \\
6 & $-0,002$ & $-0,001$ & 0,700 & {$[0,995]$} \\
7 & $-0,022$ & $-0,022$ & 0,895 & {$[0,996]$} \\
8 & 0,008 & 0,007 & 0,919 & {$[0,999]$} \\
9 & $-0,036$ & $-0,038$ & 1,450 & {$[0,998]$} \\
10 & 0,025 & 0,025 & 1,696 & {$[0,998]$} \\
11 & 0,020 & 0,020 & 1,860 & {$[0,999]$} \\
12 & $-0,003$ & $-0,002$ & 1,864 & {$[1,000]$} \\
\hline
\end{tabular}


Como pode ser verificado, tendo por base os resultados contidos nas Tabelas 5 e 6 , nenhuma ACF, PACF ou estatística $Q$ de Ljung e Box da série dos resíduos das variações dos logaritmos dos índices estudados, estimados através do modelo VAR com 12 defasagens, obteve significância estatística em nível aceitável cientificamente. Esses resultados indicam que os resíduos das séries das variações dos logaritmos dos índices não apresentam correlação serial. Dessa forma, tornou-se possível estimar os modelos GARCH multivariados CCC e DCC sem que ocorram vieses nos parâmetros estimados.

Para verificar a relação empírica de transmissão de volatilidade entre os índices Small Cap e Mid-Large Cap, foi aplicado o modelo CCC, previamente referenciado neste trabalho, nas séries integrantes da pesquisa. Os resultados dos parâmetros estimados são apresentados na Tabela 7.

\begin{tabular}{|c|c|c|c|c|}
\hline $\begin{array}{c}\text { Parâme- } \\
\text { tro }\end{array}$ & Coeficiente & Erro Padrão & Teste-t & Significância \\
\hline$C(1)$ & 0,000 & 0,000 & 395,484 & 0,000 \\
\hline$C(2)$ & 0,000 & 0,000 & $-253,105$ & 0,000 \\
\hline$A(1,1)$ & 0,165 & 0,003 & 58,328 & 0,000 \\
\hline$A(1,2)$ & $-0,108$ & 0,001 & $-118,625$ & 0,000 \\
\hline$A(2,1)$ & 0,094 & 0,000 & 681,761 & 0,000 \\
\hline$A(2,2)$ & $-0,077$ & 0,000 & $-574,454$ & 0,000 \\
\hline$B(1,1)$ & 0,542 & 0,002 & 263,080 & 0,000 \\
\hline$B(1,2)$ & 0,373 & 0,001 & 297,913 & 0,000 \\
\hline$B(2,1)$ & $-0,063$ & 0,000 & $-3276,803$ & 0,000 \\
\hline$B(2,2)$ & 1,049 & 0,000 & 22403,278 & 0,000 \\
\hline$R(2,1)$ & 0,889 & 0,001 & 1123,252 & 0,000 \\
\hline \multicolumn{5}{|c|}{ Log Likelihood 2.356,233 } \\
\hline
\end{tabular}

Os resultados da Tabela 7 permitem auferir que, durante o período amostral considerado, os choques de inovação defasados afetaram a volatilidade das ações tanto das grandes como das pequenas empresas, pois os coeficientes dos parâmetros $A(1,1)$ e $A(2,2)$ apresentaram significância estatística. $O$ mesmo ocorreu com os coeficientes dos parâmetros $A(1,2)$ e $A(2,1)$, evidenciando o fato de que os índices impactaram um ao outro no período analisado.

Quanto ao efeito $G A R C H$, os parâmetros da matriz $B$ obtiveram coeficientes significativos, sem exceção. Tal resultado evidencia que, na amostra pesquisada, ambos os índices tiveram sua volatilidade condicional impactada por dias de negociação defasados. Não obstante, os resultados permitem auferir, ainda, que houve transmissão de volatilidade entre as ações de grandes e pequenas empresas de forma bilateral. Essa constatação difere da maioria dos estudos anteriormente realizados, em que o sentido da transmissão era unilateral, com as grandes ações impactando as pequenas. Os resultados também indicaram que a correlação condicional entre os dois índices foi de 0,889, o qual obteve elevada significância estatística.

Corroborando o modelo, na Figura 1 são expostos os gráficos das volatilidades estimadas, considerando-se correlação condicional constante. Como é possível observar, o índice que representa as ações das pequenas empresas obteve níveis de volatilidade superiores ao representante das ações das grandes empresas. Esse resultado está coerente com a literatura clássica de finanças, pois ações de empresas pequenas possuem menor liquidez, o que tende a elevar a oscilação dos preços. 
Figura 1. Volatilidade estimada pelo modelo CCC para os índices Small Cap (a) e Mid-Large Cap (b).

(a) Volatilidade Small Cap

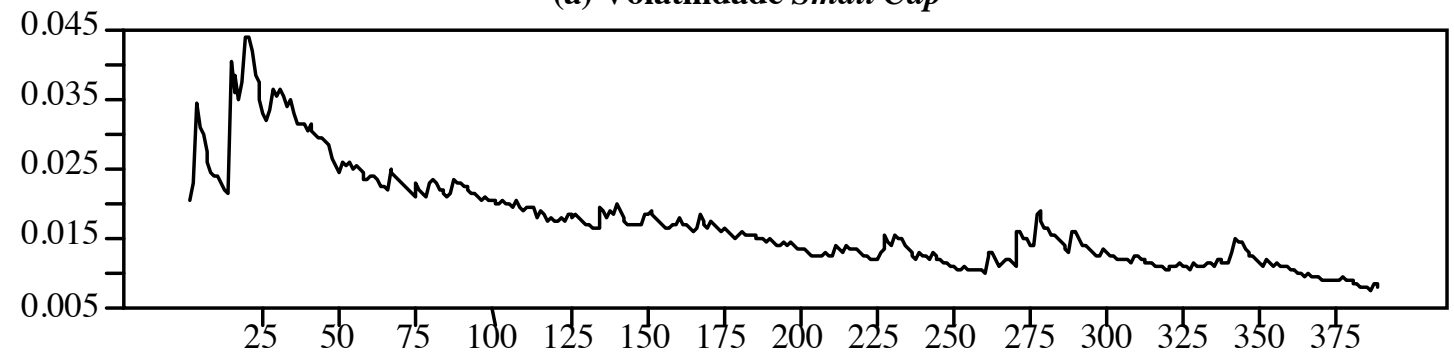

(b) Volatilidade Mid-Large Cap

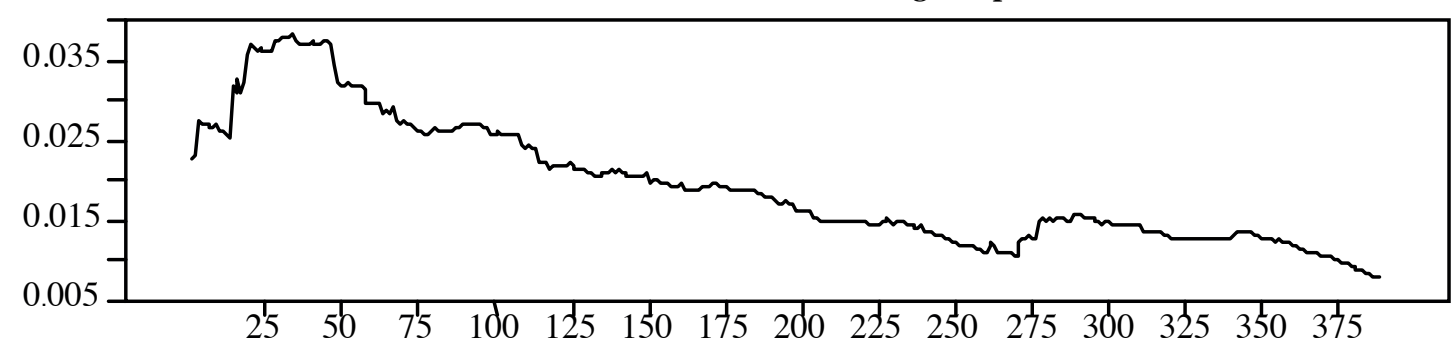

Entretanto, conforme exposto previamente no referencial teórico, o modelo CCC considera que a correlação condicional entre os ativos é constante. Tal fato não se verifica tão facilmente na prática. Dessa forma, dando sequência à análise da transmissão de volatilidade entre as ações de grandes e pequenas empresas, estimou-se o modelo $G A R C H$ multivariado com correlação condicional dinâmica. Os resultados estão expostos na Tabela 8.

Os resultados permitem concluir que os coeficientes dos parâmetros estimados para os efeitos $A R C H$ e $G A R C H$, com exceção dos coeficientes $A(1,2)$ e $B(1,2)$, obtiveram significância estatística. Tais resultados evidenciam que a volatilidade dos dois índices, no período amostral estudado, foi impactada pelos choques de inovação defasados. Não obstante, os erros quadrados defasados das ações das grandes empresas impactaram a volatilidade das pequenas. Tal relacionamento não se manteve no sentido inverso.

Tabela 8. Coeficientes estimados para o modelo GARCH multivariado supondo correlação condicional dinâmica (MV-GARCH-DCC).

\begin{tabular}{ccccc}
\hline Parâmetro & Coeficiente & Erro Padrão & Teste-t & Significância \\
\hline $\mathrm{C}(1)$ & 0,000 & 0,000 & 3,933 & 0,000 \\
$\mathrm{C}(2)$ & 0,000 & 0,000 & 2,454 & 0,014 \\
$\mathrm{~A}(1,1)$ & 0,105 & 0,040 & 2,632 & 0,008 \\
$\mathrm{~A}(1,2)$ & 0,048 & 0,053 & 0,891 & 0,373 \\
$\mathrm{~A}(2,1)$ & 0,210 & 0,009 & 24,099 & 0,000 \\
$\mathrm{~A}(2,2)$ & $-0,096$ & 0,027 & $-3,545$ & 0,000 \\
$\mathrm{~B}(1,1)$ & 0,835 & 0,051 & 16,267 & 0,000 \\
$\mathrm{~B}(1,2)$ & $-0,003$ & 0,074 & $-0,046$ & 0,963 \\
$\mathrm{~B}(2,1)$ & $-0,186$ & 0,008 & $-24,828$ & 0,000 \\
$\mathrm{~B}(2,2)$ & 1,070 & 0,028 & 37,847 & 0,000 \\
DCC(1) & 0,087 & 0,051 & 1,709 & 0,087 \\
DCC(2) & 0,884 & 0,055 & 16,163 & 0,000 \\
Shape & 6,897 & 0,575 & 12,000 & 0,000 \\
\multicolumn{5}{c}{} \\
\hline \multicolumn{5}{c}{ Log Likelihood 2.373,754 } \\
\hline
\end{tabular}


Corroborando essa análise, ainda com base nos resultados exibidos na Tabela 8, verificou-se que a volatilidade condicional de ambos os índices recebeu impacto dos dias de negociação defasados. Durante o período analisado, houve transmissão de volatilidade das ações das grandes empresas para as ações das pequenas empresas. Entretanto, essa relação não se mostrou significativa no sentido contrário.

Os resultados encontrados, com base no modelo DCC, estão coerentes com os diversos estudos referenciados na literatura de finanças sobre esse tema (MECH, 1993; MCQUEEN, PINEGAR e THORLEY, 1996; CAMPBELL, LO e MACKINLAY, 1997; GRINBLATT, TITMAN e WERMERS, 1995; CONRAD, GULTEKIN E KAUL, 1991).

Ainda com base na Tabela 8, verificou-se que, no intuito de modelar a correlação condicional por meio de uma reta, o coeficiente $\operatorname{DCC}(2)$, que representa a inclinação da mesma, obteve significância estatística no nível de $5 \%$. Tal resultado não ocorreu com o coeficiente $\mathrm{DCC}(1)$, que representa o intercepto da reta. Através da significância do parâmetro shape, auferiu-se que a correlação condicional entre os índices presentes no estudo aproximou-se de uma distribuição $t$.

Dessa forma, estão expostos na Figura 2 os gráficos acerca da volatilidade das ações das grandes e pequenas empresas, bem como sua correlação condicional, obtidos por meio do modelo DCC, com base nos dados do período pesquisado.

Figura 2. Volatilidade estimada pelo modelo DCC para os índices Small Cap (a) e Mid-Large Cap (b) e sua correlação condicional dinâmica (c).

(a) Volatilidade Small Cap

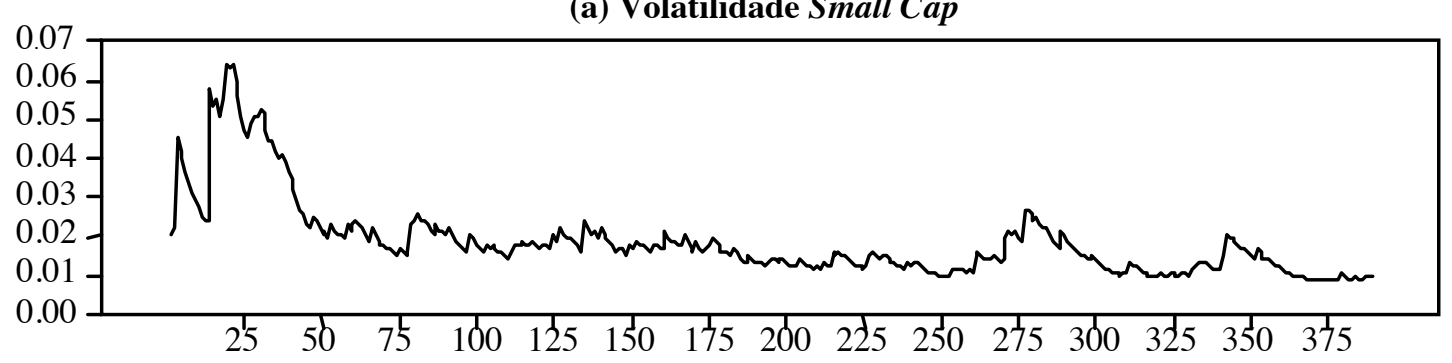

(b) Volatilidade Mid-Large Cap

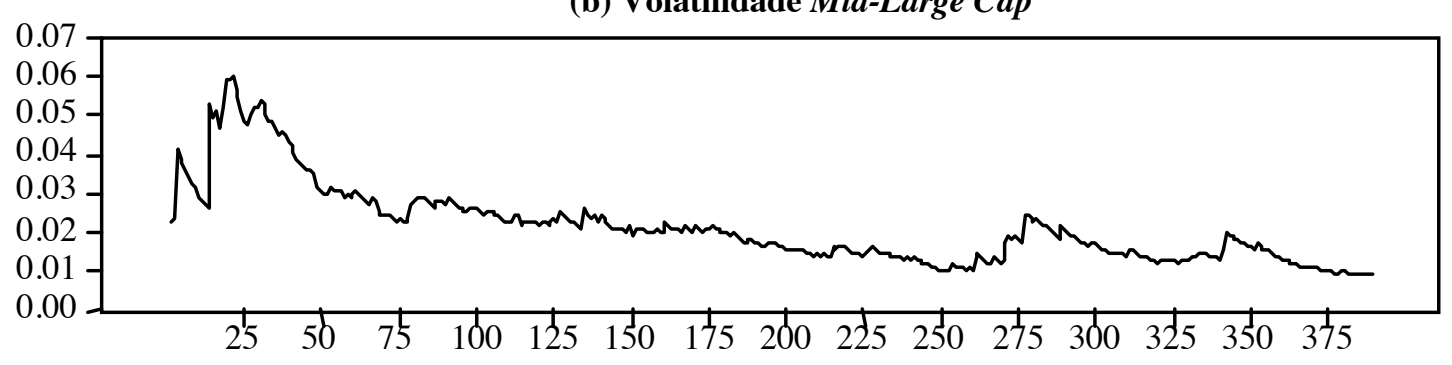

(c) Correlação Dinâmica entre Small Cap e Mid-Large Cap

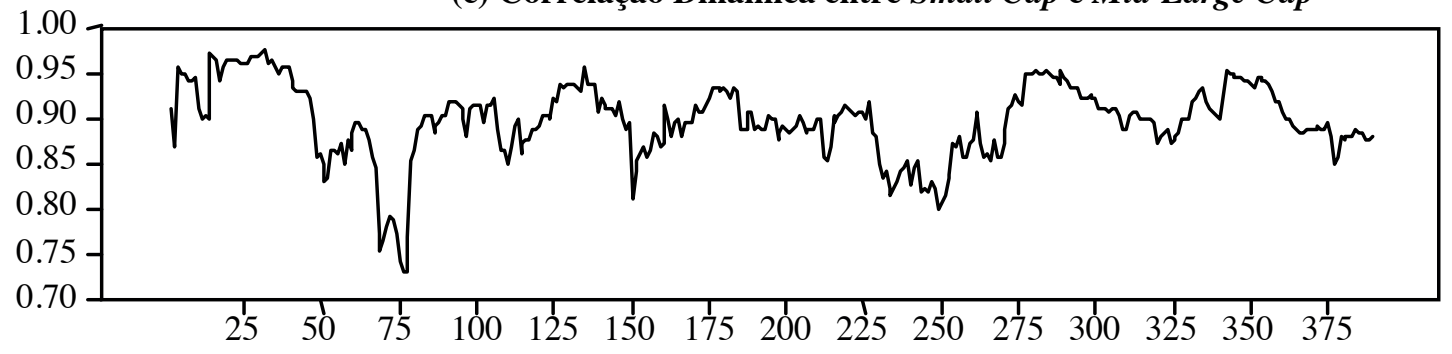




\section{CONCLUSÃO}

O presente trabalho possuiu como objetivo primordial auferir a ocorrência de transmissão de volatilidade entre ações de grandes e pequenas empresas. Para tanto, foram utilizados como representantes dessas ações os índices Small Cap e Mid-Large Cap. O método econométrico aplicado consistiu nos modelos GARCH multivariados, com correlação condicional constante (CCC) e correlação condicional dinâmica (DCC).

Após efetuar testes e procedimentos estatísticos para identificar e filtrar a dependência serial das séries das variações dos logaritmos dos índices analisados, foram estimados os modelos CCC e DCC. Os resultados obtidos por meio do modelo CCC indicaram que, durante o período estudado, ambos os índices tiveram sua volatilidade condicional impactada pelos dias de negociação defasados. Verificou-se também que, contrariando a literatura subjacente ao tema, houve transmissão bilateral de volatilidade.

Contudo, conforme referenciado no presente artigo, o modelo CCC possui limitações quanto à sua aplicação, devido à suposição de correlação condicional constante ser empiricamente utópica. Assim sendo, os resultados obtidos com o modelo DCC devem, teoricamente, representar com maior precisão a realidade. Dessa forma, verificou-se que a volatilidade condicional dos dois índices foi impactada pelos dias de negociação defasados. Esse resultado é semeIhante àquele encontrado com o modelo CCC.

Entretanto, no que tange à transmissão de volatilidade, o modelo DCC gerou resultados distintos dos obtidos por meio do modelo CCC. Tais resultados enfatizam que, durante o período estudado, houve transmissão de volatilidade das ações das grandes empresas para as ações de pequenas empresas, porém não existiu relacionamento congruente no sentido inverso. Tal diferença nos resultados obtidos entre os dois modelos foi encontrada recentemente, de forma similar, no trabalho de Khamkaew et al. (2010). Essa assimetria percebida no presente artigo é condizente com a literatura inerente ao tema e utilizada para referenciar esta pesquisa.

Como limitações do presente artigo, destacam-se: i) período amostral estudado, pois podem ocorrer variações nos resultados em diferentes períodos temporais; e ii) representação das ações das grandes e pequenas empresas, devido à utilização de índices do mercado financeiro como proxies. Para futuros estudos, sugere-se que seja auferida a transmissão de volatilidade entre diferentes setores da economia brasileira por meio de diferentes metodologias econométricas.

\section{REFERÊNCIAS}

AKAIKE, $H$. Information theory and an extension of the maximum likelihood principle. Proceedings of the $2^{\text {nd }}$ International Symposium of Information Theory, 1973.

AKAIKE, H. A new look at the statistical model identification. IEEE Transactions on automatic control, n.19, 1974.

BAUR, D. A Flexible Dynamic Correlation Model. Econometric Analysis of Financial e Economics Time Series/Part A. Advances in econometrics,
Vol. 20, 3-31. Elsevier, 2006.

BAUWENS, L.; LAURENT, S.; ROMBOUTS, J.V.K. Multivariate GARCH models: A Survey. CORE Discussion Paper No. 2003/31, 2003.

BOLLERSLEV, T. Generalized autoregressive conditional heterosdasticity. Journal of Econometrics 31, 307-327, 1986.

BOLLERSLEV, T.; WOOLBRIDGE, J. M. QuasiMaximum Likelihood Estimation and Inference 
in Dynamic Models with Time-Varying Covariances. Econometric Reviews n.11 143172, 1992.

CAMPBELL, J.; LO, A.; MACKINLAY, A. The Econometrics of Financial Markets, Princeton University Press, 1997.

CAPORIN, M.; MCALEER, M. Do We Really Need Both BEKK and DCC? A Tale of Two Multivariate GARCH Models. Working Paper, 2010. Disponível em http://papers.ssrn. com/sol3/papers.cfm ?abstract_id=1549167. Acesso em 08/09/2010.

CHAN, K.; CHAN, K. C.; KAROLYI, G. A. Intraday volatility in the stock index and stock index futures markets. Review of Financial Studies, 4, 657-684, 1991.

CONRAD, J.; GULTEKIN, M.; KAUL, G. "Asymmetric Predictability of Conditional Variances", Review of Financial Studies, vol. 4, pp. 597-622, 1991.

DARK, J.; ZHANG, X.; QU, N. Influence Diagnostics for Multivariate GARCH Processes. Journal of Time Series Analysis, vol. 31, I. 4, pp. 278-291, 2010.

DE LUCA, G.; GENTON, M. G.; LOPERFIDO, N. A Multivariate Skew-Garch Model. Econometric Analysis of Financial e Economics Time Series/ Part A. Advances in econometrics, Vol. 20, 3-31. Elsevier, 2006.

ENGLE, R. Autoregressive conditional heterosdasticity with estimates of the variance of uk inflation. Econometrica 50, 987-1007, 1982.

ENGLE, R. F.; ITO, T.; LIN, W. L. Meteor showers or heat waves? Heteroskedastic intra- daily volatility in the foreign exchange market. Econometrica, 58, pg. 525-542, 1990.

ENGLE, R. F.; SHEPPARD, K. Theoretical and Empirical properties of Dynamic Conditional Correlation Multivariate GARCH, NBER Working Paper, n.8554, 2001.
ENGLE, R.; KRONER, K. Multivariate simultaneous generalised ARCH. Econometric Theory 11, 122-150, 1995.

GOODHART, C. A. E.; O'HARA, M. High frequency data in financial markets: Issues and applications. Journal of Empirical Finance, 4:73-114, 1997.

GRINBLATT, M.; TITMAN, S.; WERMERS, R. Momentum Investment Strategies, Portfolio Performance, and Herding: A Study of Mutual Fund Behavior. American Economic Review, vol. 85, pp. 1088-1105, 1995.

HANNAN, E. J.; QUINN, B. G. The Determination of the Order of an Autoregression, Journal of the Royal Statistical Society, B, 41, 190-195, 1979.

KARMAKAR, M. Information transmission between small and large stocks in the National Stock Exchange in India: An Empirical Study, Quarterly Review of Economics and Finance, 2008.

KHAMKAEW, T.; TANSUCHAT, R.; CHANG, C. L.; MCALEER, M. Modelling Conditional Correlations in the Volatility of Asian Rubber Spot and Futures Returns. Working Paper. Center for International Research on the Japanese Economy (CIRJE) Faculty of Economics, University of Tokyo, 2010. Disponível em http://papers.ssrn.com/sol3/ papers.cfm?abstract_id=1496309. Aceso em 08/09/2010.

LJUNG, G. M.; BOX, G. E. P. On a Measure of a Lack of Fit in Time Series Models. Biometrika, n.65, pp. 297-303, 1978.

LO, A. W.; MACKINLAY, A. C. An Econometric Analysis of Nonsynchronous Trading. Journal of Econometrics, vol. 45, pp. 181-211, 1990a.

LO, A. W.; MACKINLAY, A. C. When are Constrarian Profits due to Shock Market Overreaction? Review of Financial Studies, vol. 3, pp. 175-205, 1990b. 
MCQUEEN, G.; PINEGAR, M.; THORLEY, S. Delayed Reaction to Good News and the CrossAutocorrelation of Portfolio Returns', Journal of Finance", vol. 51, No. 3, pp. 889-919, 1996.

$\mathrm{MECH}, \mathrm{T}$. Portfolio Autocorrelation. Journal of Financial Economics, vol. 34, pp. 307-44, 1993.

PETERS, T. Forecasting the covariance matrix with the DCC GARCH model. Examensarbeten $i$ matematisk statistic, 2008.

PUTINTSEVA, M. Mixture Dynamic Conditional Correlation Model. Working Paper, University of Zurich - Swiss Banking Institute (ISB); Swiss Finance Institute, 2010. Disponível em: http:// papers.ssrn.com/sol3/papers.cfm?abstract_ id=1630827. Acesso em 08/09/2010.

ROSS, S. Information and volatility: the noarbitrage martingale approach to timing and resolution irrelevancy. Journal of Finance, vol. 44, pp. 1-17, 1989.

SCHWARZ, G. Estimating the dimension of a model. Annals of statistics, n.6, 1978.

SILVENNOINEN, A.; TERÄSVIRTA, T. Multivariate GARCH models. Handbook of Financial Time Series. Springer, 2008.

TSAY, R.S. Analysis of financial time series. John Wiley \& Sons, 2005.

TSE, Y. K. A test for constant correlations in multivariate GARCH model. Journal of Econometrics, 98(1), pp. 107-127, 2000. 